Research Paper

\title{
Should All Nasopharyngeal Carcinoma with Paranasal Sinus Invasion Be Staged as T3 in the Intensity- Modulated Radiotherapy Era? A Study of 1811 Cases
}

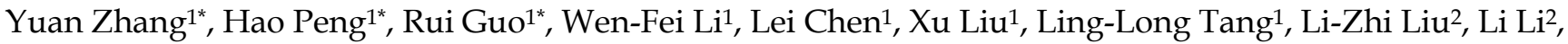 \\ Qing Liu ${ }^{3}$, Ying Sun ${ }^{1}$, Jun $\mathrm{Ma}^{1 凶}$ \\ 1. Department of Radiation Oncology, Sun Yat-sen University Cancer Center, State Key Laboratory of Oncology in South China, Collaborative Innovation \\ Center for Cancer Medicine, Canton, Guangdong Province, People's Republic of China. \\ 2. Imaging Diagnosis and Interventional Center, Sun Yat-sen University Cancer Center, State Key Laboratory of Oncology in South China, Collaborative \\ Innovation Center for Cancer Medicine, Canton, Guangdong Province, People's Republic of China. \\ 3. Department of Medical Statistics and Epidemiology, School of Public Health, Sun Yat-sen University, Canton, People's Republic of China. \\ *These authors contributed equally to this work.
}

$\square$ Corresponding author: Prof. Jun Ma, M.D., Department of Radiation Oncology, Sun Yat-sen University Cancer Center, 651 Dongfeng Road East, Canton 510060, People's Republic of China. Telephone: +86-20-87343469 Fax: +86-20-87343295 E-mail: majun2@mail.sysu.edu.cn.

(1) Ivyspring International Publisher. Reproduction is permitted for personal, noncommercial use, provided that the article is in whole, unmodified, and properly cited. See http://ivyspring.com/terms for terms and conditions.

Received: 2016.01.30; Accepted: 2016.05.18; Published: 2016.06.28

\begin{abstract}
Background: Currently, there is no uniform consensus regarding the appropriate staging for invasion of the paranasal sinuses in nasopharyngeal carcinoma (NPC). In the current AJCC staging system for NPC, paranasal sinus invasion is defined within the T3 classification. However, according to the Chinese 2008 staging system, which is also widely used in the regions where NPC is endemic in China, paranasal sinus invasion is classified as T4 disease.

Methods: Patients $(n=1811)$ with non-metastatic, histologically-proven NPC treated with intensity-modulated radiotherapy (IMRT) were retrospectively analyzed.

Results: Paranasal sinus invasion was identified in $289 / 1811$ patients (16.0\%). Multivariate analysis revealed ethmoid sinus invasion (HR, 2.889; $95 \% \mathrm{Cl}, 1.362-6.131 ; P=0.006)$ and maxillary sinus invasion (HR, 3.110; 95\% Cl, 1.439-6.721; $P=0.004$ ) were independent prognostic factors for local relapse-free survival (LRFS). T3 patients with ethmoid sinus or maxillary sinus invasion had similar 3 -year LRFS ( $83.6 \%$ vs. $92.2 \%, P=0.132)$ as T4 patients, and had poorer LRFS $(83.6 \%$ vs. $98.3 \%, P$ $=0.006$ ) than $\mathrm{T} 3$ patients with sphenoid sinus invasion alone. Also, $\mathrm{T} 3$ patients with sphenoid sinus invasion alone had similar 3-year LRFS ( 98.3 vs. $96.4 \%, P=0.391)$ as T3 patients without paranasal sinus invasion, and a trend toward higher LRFS $(98.3 \%$ vs. $92.2 \%, P=0.065)$ than T4 patients.

Conclusion: In patients underwent IMRT, tumors with ethmoid sinus or maxillary sinus invasion had a higher risk of local failure than those with sphenoid sinus invasion alone. Sphenoid sinus invasion alone should be classified as T3 disease and ethmoid sinus or maxillary sinus involvement as T4 disease in the current AJCC staging system for NPC.
\end{abstract}

Key words: nasopharyngeal neoplasms; radiotherapy, intensity-modulated; paranasal sinuses; prognosis; neoplasm staging.

\section{Introduction}

Nasopharyngeal carcinoma (NPC) is endemic in China, where over 33000 new cases were diagnosed in 2012, which represented $40 \%$ of new NPC cases worldwide [1]. Radiotherapy is the primary treatment modality for NPC [2, 3]. Staging can provide important information when formulating treatment plans and estimating survival in NPC [4]. According to the current TNM staging system, T classification is 
based on the anatomical extent of the primary tumor and has been proven to reflect the risk of local failure $[4,5]$. In particular, involvement of the paranasal sinuses, which include the sphenoid sinus, ethmoid sinus, maxillary sinus and frontal sinus, are important landmarks of local tumor extension surrounding the nasopharynx in the anterior and superior directions. The reported incidence of paranasal sinus invasion is as high as $23.6 \%-27.0 \%$ [6-9].

However, there is no uniform consensus regarding staging of paranasal sinus invasion in current staging systems. In the 7th edition of the American Joint Committee on Cancer (AJCC) TNM staging system published in 2009, paranasal sinus involvement is one of the staging criteria for T3 disease [4]. However, the Chinese 2008 staging system is also commonly used in Southern China - where the incidence of NPC is significantly higher than other regions of the world - and classifies involvement of the paranasal sinuses as T4 disease [10].

Another important issue is whether involvement of the four paranasal sinuses, which are different distances from the nasopharynx and adjacent to different critical organs, should be included in the same $\mathrm{T}$ classification. Tian et al. investigated a series of patients who were mainly $(80.2 \%)$ treated by two-dimensional radiotherapy (2D-CRT), and reported no significant differences in the survival outcomes for patients with sphenoid sinus invasion alone and patients with involvement of the ethmoid sinus or maxillary sinus [9]. However, in the last few years, intensity-modulated radiotherapy (IMRT) has gradually replaced 2D-CRT as the primary radiotherapy technique for NPC. IMRT provides excellent target conformity and dose coverage. A multicenter phase II trial and large series from a number of different centers have reported IMRT provides superior local control and long-term survival [11-16]. Therefore, the prognostic significance of paranasal sinus invasion may have altered in the IMRT era. In a study to establish a prognostic scoring model for loco-regional control in patients with NPC treated with IMRT, Tao et al. reported that involvement of the ethmoid sinus or maxillary sinus was associated with a significantly higher risk of loco-regional relapse compared to sphenoid sinus invasion [8]. However, to the best of our knowledge, the appropriate staging for paranasal sinus invasion has not been comprehensively assessed in the IMRT era. Therefore, we analyzed a large cohort of MRI-staged patients treated with IMRT in the endemic area to investigate the optimal classification for paranasal sinus invasion in the current AJCC staging system for NPC.

\section{Patients and Methods}

\section{Patients}

All 1811 patients with newly-diagnosed, histologically-proven, non-distant metastatic NPC who completed a full course of IMRT at our institution between December 2009 and February 2012 were retrospectively reviewed. All patients signed an informed consent approved by the ethics committees of our cancer center, and this study was carried out in accordance with the approved ethical guidelines and regulations. The median age of the entire cohort was 45.0 years (range, $14-78$ years), with a male-to-female ratio of 2.9:1.

All patients underwent a pretreatment evaluation including a complete patient history, physical examination, hematology and biochemistry profiles, MRI of the neck and nasopharynx, chest radiography, abdominal sonography and whole body bone scan using single photon emission computed tomography (SPECT). Furthermore, positron emission tomography-computed tomography (PET-CT) was performed on 528/1811 (29.2\%) patients. Medical records and imaging studies were analyzed retrospectively, and all patients were staged according to the 7th edition of the International Union against Cancer/American Joint Committee on Cancer (UICC/AJCC) system [4]. The characteristics of the patients analyzed in this study are shown in Table 1.

\section{Imaging}

The region from the suprasellar cistern to the suprasternal notch were examined with a 1.5-T MRI system (Signa CV/i; General Electric Healthcare, Chalfont St. Giles, United Kingdom). Firstly, axial, coronal and sagittal T1- weighted (repetition time [TR]/echo time [TE], 500-600 ms/10-20 ms), and axial T2-weighted (TR/TE, 4,000-6,000/95-110 ms) fast spin-echo MR images were obtained. Secondly, gadopentetate dimeglumine $(0.1 \mathrm{mmol} / \mathrm{kg}$; Magnevist, Schering, Berlin, Germany) was injected intravenously. Then axial and sagittal spin-echo T1-weighted and coronal spin-echo T1-weighted fat-suppressed sequences were obtained. Further details regarding MR imaging techniques in our center have been reported previously [9, 15]. All MRI scans were reviewed jointly by two radiologists with more than 10 years' experience in head and neck cancer MRI. Paranasal sinuses involvement was diagnosed using the following criteria: (1) extension of primary tumor into the sinus cavity with sinus wall destruction; and (2) the lesion in the sinuses had the same signal intensity characteristics as the primary tumor. [9] The anatomical sites of the paranasal sinuses are shown in Figure 1. 
Table 1. Clinicopathological characteristics of 1811 patients with nasopharyngeal carcinoma stratified by the presence or absence of paranasal sinus invasion.

\begin{tabular}{|c|c|c|c|}
\hline Characteristic & $\begin{array}{c}\text { No. of } 1522 \\
\text { patients without } \\
\text { paranasal sinus } \\
\text { invasion }\end{array}$ & $\begin{array}{c}\text { No. of } 289 \\
\text { patients with } \\
\text { paranasal sinus } \\
\text { invasion }\end{array}$ & $P$ \\
\hline Gender & & & 0.29 \\
\hline Male & 1128 & 223 & \\
\hline Female & 394 & 66 & \\
\hline Age (years) & & & 0.12 \\
\hline$<50$ & 1056 & 187 & \\
\hline$\geq 50$ & 466 & 102 & \\
\hline Histological type a & & & 0.22 \\
\hline $\begin{array}{l}\text { Keratinizing squamous cell } \\
\text { carcinoma }\end{array}$ & 7 & 3 & \\
\hline Non-keratinizing carcinoma & 1515 & 286 & \\
\hline Chemotherapy & & & $<0.001$ \\
\hline Yes & 1276 & 276 & \\
\hline No & 246 & 13 & \\
\hline T-category b & & & $<0.001$ \\
\hline $\mathrm{T} 1$ & 326 & 0 & \\
\hline $\mathrm{T} 2$ & 283 & 0 & \\
\hline $\mathrm{T} 3$ & 770 & 95 & \\
\hline $\mathrm{T} 4$ & 143 & 194 & \\
\hline N-category b & & & 0.001 \\
\hline N0 & 277 & 31 & \\
\hline N1 & 881 & 181 & \\
\hline N2 & 218 & 58 & \\
\hline N3a & 26 & 6 & \\
\hline $\mathrm{N} 3 \mathrm{~b}$ & 120 & 13 & \\
\hline Stage-group ${ }^{b}$ & & & $<0.001$ \\
\hline I & 99 & 0 & \\
\hline II & 382 & 0 & \\
\hline III & 770 & 87 & \\
\hline IVa & 151 & 189 & \\
\hline $\mathrm{IVb}$ & 120 & 13 & \\
\hline
\end{tabular}

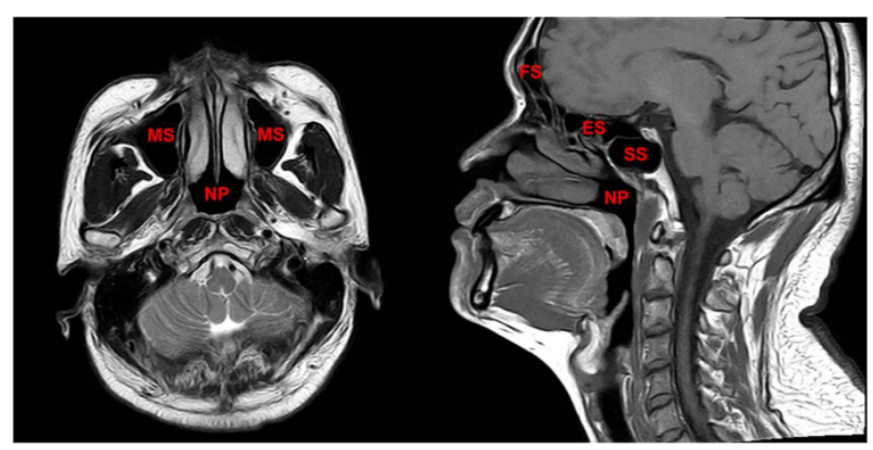

Figure 1: The paranasal sinuses, which include the sphenoid sinus, ethmoid sinus, maxillary sinus and frontal sinus, are important anatomical structures that surround the nasopharynx in the anterior and superior directions. Abbreviations: $\mathrm{SS}=$ sphenoid sinus; $\mathrm{ES}=$ ethmoid sinus; $\mathrm{MS}=$ maxillary sinus; $\mathrm{FS}$ = frontal sinus; $\mathrm{NP}=$ nasopharynx.

\section{Treatment}

The nasopharyngeal and neck tumor volumes of all patients were treated using radical radiotherapy based on IMRT for the entire treatment course. Target volumes were delineated slice-by-slice on treatment planning CT scans using an individualized delineation protocol that complies with International Commission on Radiation Units and Measurements reports 50 and 62 . The prescribed doses were $66-72$ Gy at 2.12-2.43 Gy/fraction (with $4.5 \%$ of patients over $2.33 \mathrm{~Gy} /$ fraction for they enrolled in a clinical trial in our center) to the planning target volume (PTV) of the primary gross tumor volume (GTVnx), 64-70 Gy/ 28-33 fractions to the PTV of the GTV of the involved lymph nodes (GTVnd), 60-63 Gy/ 28-33 fractions to the PTV of the high-risk clinical target volume (CTV1), and 54-56 Gy/ 28-33 fractions to the PTV of the low-risk clinical target volume (CTV2). The plan could be accepted if the prescribed dose encompassed at least $95 \%$ of the target volume. All targets were treated simultaneously using the simultaneous integrated boost technique; other details of the techniques used at our center have previously been reported [12].

During the study, institutional guidelines recommended radiotherapy alone for stage I and concurrent chemoradiotherapy \pm neoadjuvant /adjuvant chemotherapy for stage II to IVB. In total, 93.5\% (1244/1330) of patients with stage III-IVB NPC received concurrent chemoradiotherapy \pm neoadjuvant/adjuvant chemotherapy. When possible, salvage treatments (intracavitary brachytherapy, surgery or chemotherapy) were provided in documented relapse or persistent disease.

\section{Follow-up}

Each patient was assessed for treatment response and toxicity every week during treatment, every 3 months during the first 2 years after radiotherapy and every 6 months during years 3-5. Patients with residual or recurrent local disease underwent biopsy to confirm malignancy. CT, MRI, or PET-CT was used for diagnosis of residual/ recurrent disease if biopsy was not feasible. Additional tests were ordered when indicated to evaluate for local or distant failure.

\section{Statistical analysis}

Statistical analyses were performed using SPSS version 22.0 (IBM Corporation, Armonk, NY, USA). The outcome of interest was local relapse-free survival (LRFS). LRFS was calculated from the first day of treatment to the first local relapse. Survival rates were calculated using the Kaplan-Meier method and compared using the log-rank test [17]. Multivariate analyses with the Cox proportional hazards model were used to calculate hazard ratios (HRs), 95\% confidence intervals (CIs) and test the independent significance of different factors by backward elimination of insignificant variables [18], and included host factors (sex, age), pathological type (keratinizing squamous cell carcinoma vs. 
non-keratinizing carcinoma), chemotherapy, and tumor factors (skull base erosion, hypopharyngeal extension, orbit extension, masticator space extension, cranial nerve palsy and intracranial extension; $\mathrm{N}$ category) as covariates. Two-tailed $P$-values $<0.05$ were considered statistically significant.

\section{Results}

\section{Incidence of paranasal sinus invasion}

The incidence of paranasal sinus invasion was $16.0 \%$ (289 of 1811 patients), with invasion of the sphenoid sinus, ethmoid sinus and maxillary sinus observed in $271(15.0 \%), 89(4.9 \%)$ and $76(4.2 \%)$ of the 1811 patients, respectively. No patients had frontal sinus invasion. Of the 271 patients with sphenoid sinus invasion, 104 (38.1\%) also had ethmoid sinus or maxillary sinus invasion. Of the 89 patients with ethmoid sinus invasion and 76 patients with maxillary sinus invasion, $65(85.5 \%)$ and 79 (88.8\%), respectively, also had sphenoid sinus invasion. According to the T classification of the seventh edition of the AJCC staging system, of the 289 patients with paranasal sinus invasion, $95(32.9 \%)$ had T3 disease and $194(67.1 \%)$ had T4 disease. The distribution of paranasal sinus invasion by $\mathrm{T}$ classification is shown in Table 2.

Table 2. Distribution of paranasal sinus invasion in 1811 patients with nasopharyngeal carcinoma by $\mathrm{T}$ classification.

\begin{tabular}{llllllll}
\hline & $\begin{array}{l}\text { SS+, } \\
\text { MS-, ES- }\end{array}$ & $\begin{array}{l}\text { MS+, } \\
\text { SS-, ES- }\end{array}$ & $\begin{array}{l}\text { ES+, } \\
\text { SS-, } \\
\text { MS- }\end{array}$ & $\begin{array}{l}\text { SS+, } \\
\text { MS+, } \\
\text { ES- }\end{array}$ & $\begin{array}{l}\text { SS+, } \\
\text { ES+, } \\
\text { MS- }\end{array}$ & $\begin{array}{l}\text { MS+, } \\
\text { ES+, } \\
\text { SS- }\end{array}$ & $\begin{array}{l}\text { SS+, } \\
\text { MS+, } \\
\text { ES+ }\end{array}$ \\
\hline T3 & 63 & 7 & 4 & 4 & 9 & 2 & 6 \\
T4 & 104 & 1 & 3 & 21 & 30 & 1 & 34 \\
\hline \multicolumn{7}{l}{ Abbreviations: SS = sphenoid sinus; ES = ethmoid sinus; MS = maxillary sinus. }
\end{tabular}

Table 3. Multivariate analysis of prognostic factors in 1811 patients with nasopharyngeal carcinoma.

\begin{tabular}{|c|c|c|c|}
\hline Endpoint & Variable & HR (95\% CI) & $P$-value \\
\hline \multirow[t]{3}{*}{$\begin{array}{l}\text { Local } \\
\text { recurrence-free } \\
\text { survival }\end{array}$} & $\begin{array}{l}\text { Histological type } \mathrm{b} \text { (keratinizing } \\
\text { squamous cell carcinoma vs. } \\
\text { non-keratinizing carcinoma) }\end{array}$ & $\begin{array}{l}7.242 \\
(1.768-29.662)\end{array}$ & 0.006 \\
\hline & Ethmoid sinus invasion & $\begin{array}{l}2.889 \\
(1.362-6.131)\end{array}$ & 0.006 \\
\hline & Maxillary sinus invasion & $\begin{array}{l}3.110 \\
(1.439-6.721)\end{array}$ & 0.004 \\
\hline \multirow[t]{4}{*}{$\begin{array}{l}\text { Overall } \\
\text { survival }\end{array}$} & Age $(<50$ vs. $\geq 50)$ & $\begin{array}{l}1.946 \\
(1.359-2.785)\end{array}$ & $<0.001$ \\
\hline & N classification c (N0-1 vs. N2-3) & $\begin{array}{l}3.961 \\
(2.760-5.685)\end{array}$ & $<0.001$ \\
\hline & Skull base invasion & $\begin{array}{l}1.946 \\
(1.217-3.111)\end{array}$ & 0.005 \\
\hline & Maxillary sinus extension & $\begin{array}{l}2.614 \\
(1.455-4.695)\end{array}$ & 0.001 \\
\hline
\end{tabular}

Abbreviations: $\mathrm{HR}=$ hazard ratio; $\mathrm{CI}=$ confidence interval.

a $P$-values were calculated using an adjusted Cox proportional hazards model.

b According to the 2005 World Health Organization classification of tumors.

cAccording to the 7th UICC/AJCC staging system.

\section{Prognosis of patients with paranasal sinus invasion}

The median follow-up period was 38.3 months for the entire cohort (range, 1.3-60.2 months). For patients alive at last follow-up, $0.7 \%(14 / 1811)$ and $2.0 \%(36 / 1811)$ had a follow-up time of less than 12 and 24 months, respectively. A total of 79/1811 (4.4\%) patients developed local failure. The 3-year LRFS rate for the entire cohort was $95.7 \%$.

We explored the prognostic value of sphenoid sinus, ethmoid sinus and maxillary sinus invasion in NPC. Univariate analysis identified sphenoid sinus, ethmoid sinus and maxillary sinus invasion as prognostic factors for 3-year LRFS (91.2\% vs. 96.5\%; $80.1 \%$ vs. $96.5 \%$; and $78.5 \%$ vs. $96.5 \%$, respectively, all $P<0.001$ ), with better outcomes in patients without sphenoid sinus, ethmoid sinus or maxillary sinus invasion, respectively. The corresponding Kaplan-Meier survival curves are shown in Figure 2.

Multivariate analysis was performed to adjust for confounding factors. Both ethmoid sinus invasion (HR, 2.889; 95\% CI, 1.362-6.131; $P=0.006)$ and maxillary sinus invasion (HR, 3.110; 95\% CI, 1.439-6.721; $P=0.004$ ) were found to be independent prognostic factors for LRFS, while sphenoid sinus invasion had no effect on local failure (HR, 0.753; 95\% CI, .338-1.673; $P=0.49$ ).

\section{Grading and staging categories for paranasal sinus involvement}

Due to the proximity of the floor of the sphenoid sinus to the roof of the nasopharynx, and as the majority of patients with maxillary sinus or ethmoid sinus invasion also had sphenoid sinus invasion, we classified paranasal sinus invasion into two grades: grade 1 , sphenoid sinus invasion alone $(n=167)$; and grade 2 , ethmoid sinus or maxillary sinus invasion with or without sphenoid sinus invasion $(n=122)$. Significant differences in 3-year LRFS were observed between grade 1 and grade $2(97.4 \%$ vs. $83.1 \%, P<$ 0.001). Moreover, the grade of paranasal sinus invasion retained independent prognostic value for local failure in multivariate analyses (HR, 2.010; 95\% CI, 1.526-2.649; $P<0.001$ ).

Next, to investigate the significance of paranasal sinus invasion in NPC with respect to the seventh AJCC staging system, we divided patients with T3-4 disease into four subgroups as follows: group 1, T3 disease without paranasal sinus invasion $(n=770)$; group 2, T3 disease with sphenoid sinus invasion alone $(n=63)$; group 3 , T3 disease with ethmoid sinus or maxillary sinus invasion $(n=32)$; and group 4 , T4 disease $(n=337)$. Kaplan-Meier survival curves for these four subgroups were shown in Figure 3. T3 patients with ethmoid sinus or maxillary sinus 
invasion had no significant differences in 3-year LRFS (83.6\% vs. $92.2 \%, P=0.13$ ) compared with T4 patients, and had an increased risk of local failure compared to T3 patients without paranasal sinus invasion $(83.6 \%$ vs. $96.4 \%, P<0.001)$. However, T3 patients with sphenoid sinus invasion alone had similar 3-year LRFS (98.3 vs. $96.4 \%, P=0.39$ ) to T3 patients without paranasal sinus invasion, and demonstrated a trend towards higher 3-year LRFS than T4 patients (98.3\% vs. $92.2 \%, P=0.06)$.
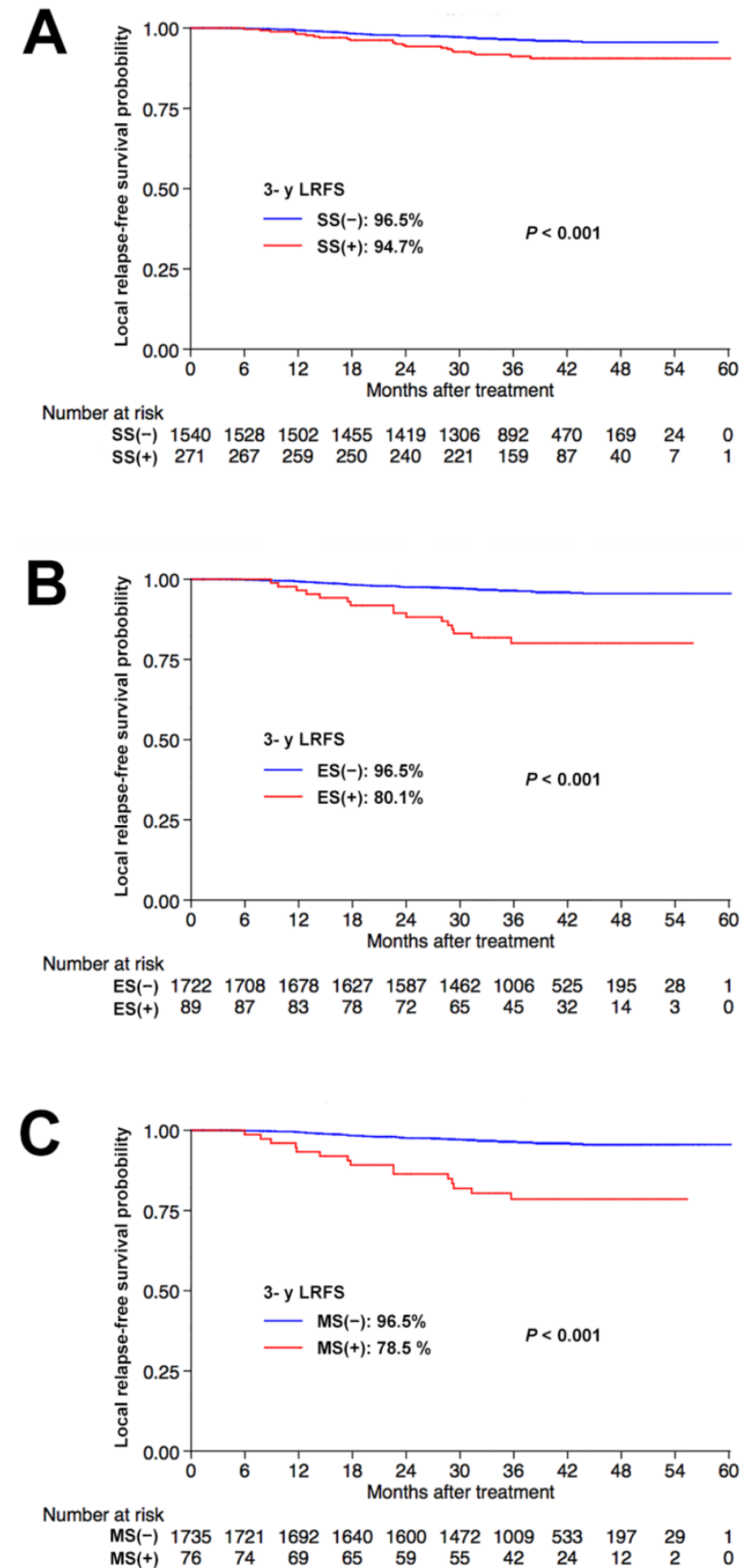

Figure 2: Kaplan-Meier local relapse-free survival curves for patients with NPC stratified by involvement of the sphenoid sinus (A), ethmoid sinus (B), and maxillary sinus $(\mathbf{C})$, respectively. Abbreviations: $3-y=3$-year; LRFS = local relapse-free survival; $\mathrm{SS}=$ sphenoid sinus; $\mathrm{ES}=$ ethmoid sinus; $\mathrm{MS}=$ maxillary sinus.

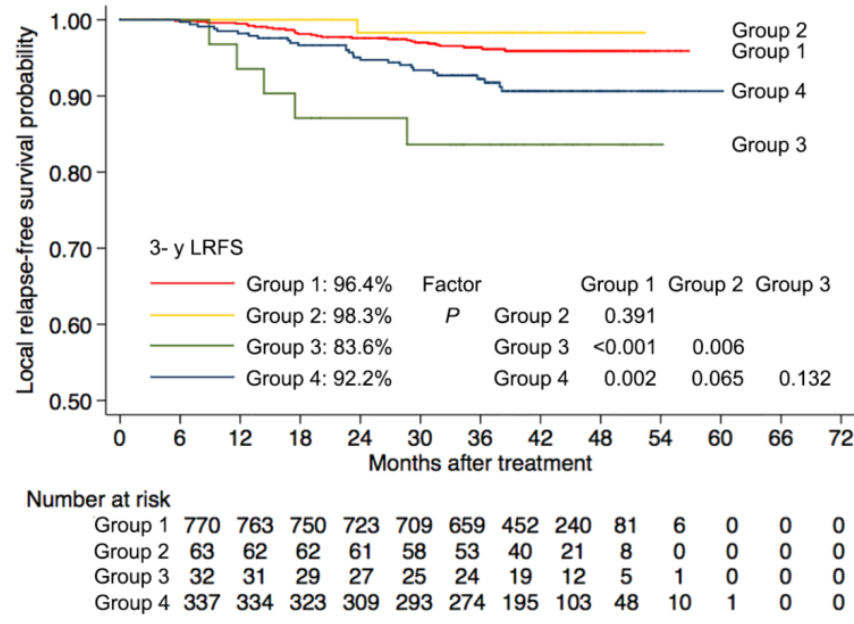

Figure 3: Probability of local relapse-free survival for patients with T3 disease and patients with T4 disease according to the current AJCC staging system. Group 1 = patients with T3 disease without paranasal sinus invasion, Group 2 = patients with T3 disease with sphenoid sinus invasion alone, Group 3 = patients with T3 disease with ethmoid sinus or maxillary sinus invasion and Group $4=$ patients with T4 disease. 3-y = 3-year; LRFS = local relapse-free survival.

\section{Discussion}

Uniform consensus has not yet been reached on appropriate staging for invasion of the paranasal sinuses. In the current edition of the AJCC staging system for NPC, paranasal sinus invasion is included in the T3 classification [4]. However, in the Chinese 2008 staging system, which is widely used in China where NPC is endemic, paranasal sinus invasion is classified as T4 disease [10]. Controversy also exists whether the involvement of different paranasal sinuses should be included in the same $T$ classification. Tao et al. reported that involvement of the ethmoid sinus or maxillary sinus were associated with a higher risk of loco-regional relapse compared to involvement of the sphenoid sinus in patients with NPC treated with IMRT [8]. Thus, staging for involvement of the paranasal sinuses in NPC requires further investigation.

In this study, we retrospectively analyzed a large cohort of patients treated with IMRT and found that involvement of the ethmoid sinus or maxillary sinus was associated with a higher risk of local failure than sphenoid sinus invasion alone. T3 disease with ethmoid sinus or maxillary sinus invasion had similar LRFS rate as T4 disease, while the LRFS rate for T3 disease with involvement of the sphenoid sinus alone were comparable to T3 disease without paranasal sinus invasion. Thus, we suggest that involvement of different paranasal sinuses should be grouped into different $\mathrm{T}$ classifications; patients with sphenoid sinus invasion alone should be classified as T3 disease whereas patients with involvement of the ethmoid 
sinus or maxillary sinus should be classified as $\mathrm{T} 4$ disease.

In the fifth edition of the AJCC staging system for NPC published in 1997, paranasal sinus invasion was defined as T3 disease [19], and this classification remained in the sixth and current seventh edition [4, 20]. However, imaging and treatment techniques for NPC have undergone tremendous changes in the last two decades. MRI has been proven to be superior to computed tomography (CT) for assessing the tumor extent and has become the imaging modality of choice [21-23]. Additionally, radiotherapy techniques have evolved from 2D-CRT to IMRT [11-16]. Thus, the staging criteria - including paranasal sinus invasion established in patients diagnosed and treated using older technologies need to be reassessed in the new era. To the best of our knowledge, this is the first study to assess the staging of paranasal sinus invasion in patients with NPC treated with IMRT; this study may provide useful information for future revisions of the current AJCC staging system.

Previous studies have demonstrated the predictive role of tumor volume for treatment outcome in NPC. Patients with a larger primary tumor volume were reported to have an increased risk of local failure [24, 25]. Due to the proximity of the floor of the sphenoid sinus to the roof of the nasopharynx, and the fact the majority of patients with ethmoid sinus (85.5\%) or maxillary sinus invasion $(88.8 \%)$ also had sphenoid sinus invasion, it is reasonable to speculate that the primary tumor volume of patients with sphenoid sinus invasion alone might be smaller than that of patients with ethmoid sinus or maxillary sinus invasion. Therefore, tumor volume may be an intrinsic factor that explains why patients with sphenoid sinus invasion have a more favorable outcome than patients with ethmoid sinus or maxillary sinus invasion.

In terms of the prevalence of paranasal sinus invasion, sphenoid sinus invasion had the highest rate $(15 \%)$, followed by ethmoid sinus invasion $(4.9 \%)$ and maxillary sinus invasion (4.2\%). As local disease spreads in a stepwise manner from proximal to distal sites and the sphenoid sinus borders the roof of the nasopharynx, sphenoid sinus invasion has the highest rate of incidence, and invasion of more distal sites such as the ethmoid sinus or maxillary sinus frequently occurs simultaneously to sphenoid sinus invasion ( $85.5 \%$ and $88.8 \%$, respectively). Compared to the respective rates of $21-27 \%, 5-9 \%$, and $4 \%-14 \%$ reported in other studies [6-9], the rate of sphenoid sinus invasion was lower in the current study. Instead of a genuine imbalance in patient composition among these large cohorts, it is more likely that variations in the diagnostic criteria for paranasal sinus invasion used by radiologists may explain this inconsistency. While there should be minimal discrepancy in the diagnosis of gross paranasal sinus invasion, subtle invasion of the paranasal sinus is not uncommon, and may pose both a diagnostic challenge and a source of variation between different centers or individual radiologists [26].

It should be stressed this study has some limitations. Firstly, there were unavoidable biases due to the retrospective nature of the study. Secondly, as $46.4 \%(840 / 1811)$ of patients underwent induction chemotherapy before radiotherapy, data on the primary tumor volume was not available. Thus, we can only speculate that a smaller tumor volume may be the factor underlying the more favorable outcome of patients with sphenoid sinus alone. Thirdly, this study only included patients from the endemic region in China, of whom only $2 \%$ had keratinizing disease (WHO type I), which accounts for up to $67 \%$ of cases of NPC in western countries [27]. Thus the results of the current study need to be confirmed in further studies of other cohorts from different regions before they can be generalized to all patients with NPC.

In conclusion, patients with ethmoid sinus or maxillary sinus invasion had a higher risk of local failure than those with sphenoid sinus invasion alone. Patients with sphenoid sinus invasion alone should be classified with T3 disease and those with involvement of the ethmoid sinus or maxillary sinus as T4 disease in the current AJCC staging system for NPC.

\section{Acknowledgements}

This work was supported by grants from the Key Laboratory Construction Project of Guangzhou City, China (No.121800085), the Health \& Medical Collaborative Innovation Project of Guangzhou City, China (201400000001), the Sun Yat-sen University Clinical Research 5010 Program (No. 2012011), the National Science \& Technology Pillar Program during the Twelfth Five-year Plan Period (2014BAI09B10), Science and Technology Project of Guangzhou City, China (No. 14570006), and the Planned Science and Technology Project of Guangdong Province (No. 2013B020400004).

\section{Conflict of Interest}

We declare that we have not conflict of interest.

\section{References}

1. IARC. GLOBOCAN 2012: Estimated Cancer Incidence, Mortality and Prevalence Worldwide in 2012. 2012.

2. Chan AT. Nasopharyngeal carcinoma. Annals of oncology : official journal of the European Society for Medical Oncology / ESMO. 2010; 21 Suppl 7: vii308-12.

3. Lee AW, Lin JC, Ng WT. Current management of nasopharyngeal cancer. Seminars in radiation oncology. 2012; 22: 233-44. 
4. Edge SB, Compton CC. The American Joint Committee on Cancer: the 7th edition of the AJCC cancer staging manual and the future of TNM. Annals of surgical oncology. 2010; 17: 1471-4.

5. Pan J, Xu Y, Qiu S, Zong J, Guo Q, Zhang Y, et al. A Comparison Between the Chinese 2008 and the 7th Edition AJCC Staging Systems for Nasopharyngeal Carcinoma. American journal of clinical oncology. 2013.

6. Chong VF, Fan YF, Khoo JB. Computed tomographic and magnetic resonance imaging findings in paranasal sinus involvement in nasopharyngeal carcinoma. Annals of the Academy of Medicine, Singapore. 1998; 27: 800-4.

7. King AD, Lam WW, Leung SF, Chan YL, Teo P, Metreweli C. MRI of local disease in nasopharyngeal carcinoma: tumour extent vs tumour stage. The British journal of radiology. 1999; 72: 734-41.

8. Tao CJ, Liu X, Tang LL, Mao YP, Chen L, Li WF, et al. Prognostic scoring system for locoregional control among the patients with nasopharyngeal carcinoma treated by intensity-modulated radiotherapy. Chinese journal of cancer. 2013; 32: 494-501.

9. Tian L, Li YZ, Mo YX, Liu LZ, Xie CM, Liang XX, et al. Nasopharyngeal carcinoma with paranasal sinus invasion: the prognostic significance and the evidence-based study basis of its T-staging category according to the AJCC staging system. BMC cancer. 2014; 14: 832.

10. Mao YP, Li WF, Chen L, Sun Y, Liu LZ, Tang LL, et al. [A clinical verification of the Chinese 2008 staging system for nasopharyngeal carcinoma]. Ai zheng = Aizheng = Chinese journal of cancer. 2009; 28: 1022-8.

11. Kam MK, Teo PM, Chau RM, Cheung KY, Choi PH, Kwan WH, et al. Treatment of nasopharyngeal carcinoma with intensity-modulated radiotherapy: the Hong Kong experience. International journal of radiation oncology, biology, physics. 2004; 60: 1440-50.

12. Lai SZ, Li WF, Chen L, Luo W, Chen YY, Liu LZ, et al. How does intensity-modulated radiotherapy versus conventional two-dimensional radiotherapy influence the treatment results in nasopharyngeal carcinoma patients? International journal of radiation oncology, biology, physics. 2011; 80: 661-8

13. Lee AW, Sze WM, Au JS, Leung SF, Leung TW, Chua DT, et al. Treatment results for nasopharyngeal carcinoma in the modern era: the Hong Kong experience. International journal of radiation oncology, biology, physics. 2005; 61: 1107-16.

14. Lee N, Harris J, Garden AS, Straube W, Glisson B, Xia P, et al. Intensity-modulated radiation therapy with or without chemotherapy for nasopharyngeal carcinoma: radiation therapy oncology group phase II trial 0225. Journal of clinical oncology : official journal of the American Society of Clinical Oncology. 2009; 27: 3684-90.

15. Sun Y, Tang LL, Chen L, Li WF, Mao YP, Liu LZ, et al. Promising treatment outcomes of intensity-modulated radiation therapy for nasopharyngeal carcinoma patients with $\mathrm{N} 0$ disease according to the seventh edition of the AJCC staging system. BMC cancer. 2012; 12: 68.

16. Tham IW, Hee SW, Yeo RM, Salleh PB, Lee J, Tan TW, et al. Treatment of nasopharyngeal carcinoma using intensity-modulated radiotherapy-the national cancer centre singapore experience. International journal of radiation oncology, biology, physics. 2009; 75: 1481-6.

17. Dinse GE, Lagakos SW. Nonparametric estimation of lifetime and disease onset distributions from incomplete observations. Biometrics. 1982; 38: 921-32.

18. Cox DR. Regression models and life- tables. J R Stat Soc Ser B (Methodological). 1972; 34: 187-220.

19. Chien CR, Chen SW, Hsieh CY, Liang JA, Yang SN, Huang CY, et al. Retrospective comparison of the AJCC 5th edition classification for nasopharyngeal carcinoma with the AJCC 4th edition: an experience in Taiwan. Japanese journal of clinical oncology. 2001; 31: 363-9.

20. Mao YP, Xie FY, Liu LZ, Sun Y, Li L, Tang LL, et al. Re-evaluation of 6th edition of AJCC staging system for nasopharyngeal carcinoma and proposed improvement based on magnetic resonance imaging. International journal of radiation oncology, biology, physics. 2009; 73: 1326-34.

21. Chung NN, Ting LL, Hsu WC, Lui LT, Wang PM. Impact of magnetic resonance imaging versus $\mathrm{CT}$ on nasopharyngeal carcinoma: primary tumor target delineation for radiotherapy. Head \& neck. 2004; 26: 241-6.

22. Liang SB, Sun Y, Liu LZ, Chen Y, Chen L, Mao YP, et al. Extension of local disease in nasopharyngeal carcinoma detected by magnetic resonance imaging: improvement of clinical target volume delineation. International journal of radiation oncology, biology, physics. 2009; 75: 742-50.

23. Liao XB, Mao YP, Liu LZ, Tang LL, Sun Y, Wang Y, et al. How does magnetic resonance imaging influence staging according to AJCC staging system for nasopharyngeal carcinoma compared with computed tomography? International journal of radiation oncology, biology, physics. 2008; 72: 1368-77.

24. Guo R, Sun Y, Yu XL, Yin WJ, Li WF, Chen YY, et al. Is primary tumor volume still a prognostic factor in intensity modulated radiation therapy for nasopharyngeal carcinoma? Radiotherapy and oncology : journal of the European Society for Therapeutic Radiology and Oncology. 2012; 104: 294-9.

25. Wu Z, Zeng RF, Su Y, Gu MF, Huang SM. Prognostic significance of tumor volume in patients with nasopharyngeal carcinoma undergoing intensity-modulated radiation therapy. Head \& neck. 2013; 35: 689-94.

26. Sze H, Chan LL, Ng WT, Hung AW, Lee MC, Chang AT, et al. Should all nasopharyngeal carcinoma with masticator space involvement be staged as T4? Oral oncology. 2014; 50: 1188-95.

27. Shedd DP, Von Essen CF, Eisenberg H. Cancer of the nasopharynx in Connecticut, 1935 through 1959. Cancer. 1967; 20: 508-11. 